\title{
Special session organized by D. De Wrachien \& M. A. Lenzi
}

\section{Scope aims and topics}

The terms resource and natural disaster are a reflection of mankind's perception of natural systems. When man recognises a natural system as useful, it becomes a resource whereas if it is potentially harmful, it is seen as a danger. In the absence of human settlement, however, there are neither resources nor dangers, but merely natural systems [1].

In this context a disaster can be defined as an event where damage exceeds the capacity of the affected society to recover by its own means. This implies that there are local disasters that can be dealt with by regional help and that there are regional or even national disasters that require national or international help. This definition does not implicitly include the terms "frequent" or "exceptional" but merely implies that the size of a disaster is related to the specific society affected and underlines the fact that the weaker are usually the most severely affected by natural disasters [2]. Water-forest related natural disasters - to which the large woody debris accumulations (LWD), debris and hyper-concentrated flows belong - are mainly the result of physical processes, which cause disasters when they interact with human activities. The mitigation of such phenomena could, therefore, be achieved by reducing this interaction, by altering the natural system, the human system of both.

The trend of increase in the environmental and economic impacts of natural disasters, and in particular the water-forest related ones, has continued during the past half century. Over the last 30 years, although the number of lives lost by natural disasters has declined, the number of people affected and estimated economic losses have been steadily increasing.

LWD, debris and hyper-concentrated flows result from the interaction of hydrological processes with geological process and are triggered when soils get saturated and the stability of the slope is no longer maintained. These flows are among the most destructive of all water-related disasters. They mainly affect mountain areas in a wide range of morphoclimatic environments and in recent years have attracted more a more attention from the scientific and professional 
communities and concern from the public awareness, due to the increasing frequency with which they occur and the death toll they claim.

Higher population pressures on natural resources in hazard-prone areas and development of activities that have the potential to increase the magnitude of hazard call for improvements in the criteria used to identify debris flow risk areas and to design prevention and mitigation measures. There is need to take into account risks emanating from these events into the development process in general and forest, land and water resources management in particular. Sustainable development should take account of hydrological variability through appropriate risk management policies in all climatic zones and hydrological regimes. Integrated forest, land and water resources management should incorporate risk management principles in dealing with extreme hydrological events like debris and hyper-concentrated flows.

The identification of effective procedures aimed at evaluating the probability of extreme hydrological events producing socio-economic damages to human settlements has turned out to be an essential component of the forest, land and water planning and management process.

It is worth noting that, whilst in the past the concept of risk was primarily intended as a measure of the probability of a system's failure, it has assumed nowadays a more complex meaning. The risk should be, in fact, considered as a combination of both the probability and the magnitude of the consequence (e.g. the effect) of a system's failure and, therefore, as the expectation of the consequence, taking into account all significant hazards and all significant mechanisms of failure [3].

In this context, the object of risk analysis changes too, depending on both the system to be considered and the goals to be pursued. The risk analysis procedure should, therefore, consist of two different and consequential phases: a first phase aimed at classifying the object of the analysis and at defining the variables on which the risk depends, and a second phase aimed at specifying the conditions and the modes of the system failure.

This concept leads to a new integrated risk management approach which comprises the systematic process, administrative decisions, organisation, operational skills and abilities to implement policies, strategies, and coping capacities of the society and communities to lessen the impact of natural hazards and related environmental disasters [4].

This approach provides measures for preventing a hazard turning into a natural disaster and consists of systematic actions in a cycle of preparedness, response and recovery that should form part of an integrated forest, land and water resources management procedure. Preparedness consists of preventive and precautionary measures to prepare for an event before it occurs. It aims at minimizing the effect of development activities on accentuating the magnitude of hazards, reducing the exposure to natural hazards and minimising the socioeconomic vulnerability of people and material assets exposed to these hazards. Response deals with measures that limit the effects of exposure to a hazard, and its duration. It mainly focuses on alerting potential affected people, rescuing victims and providing assistance in case of need. It also includes immediate 
measures to prevent further adverse impacts and provisional reconditioning of important infrastructures affected. The recovery phase aims at enabling the economic and social activities to return to normal with a minimum delay. It also involves the analysis of the disaster in order to learn lessons and ingrate corrective measures into prevention and preparedness plans. These actions are taken depending on the conditions of risk and social, economic and physical setting, with major focus on reducing the vulnerability that has to be addressed through local actions to overcome global challenges.

It is important to understand that while our planning capabilities, technological tools and methods might be adequate, our capacity to enforce these plans may be affected by political considerations and thus become closer to nonexistent. It is worth stressing that the effectiveness of the risk management cycle in reducing risks and damages depends on the political will to apply the risk management principles in development planning, the existence of a chain of well-defined institutional responsibilities and a democratic process of consultation and social control with effective governance. Well-designed prevention and mitigation measures should not only deal with day to day operation, but must also include risk analysis and suitable measures to cope with extraordinary disturbances. To this end, worst scenario emergency planning for such likely events is essential. Monitoring, forecasting and early warning play a pivotal role in the risk management cycle. Regular monitoring of elements that constitute forest-water-related hazards is crucial during the preparedness, response and reconstruction phases. Unpredictability of climatic extremes for specific regions and their extent is the vital gap in the knowledge on climate change, which needs to be addressed. Advanced scientific research should be applied in monitoring forest-water-related hazards through appropriate networks of climatic, hydrological and geological parameters.

Developments in remote sensing, satellite communication and information technology should be used for improving monitoring and developing mathematical models for forecasting and early warning of impending hazards. The challenge before the international community is to support these activities, particularly in developing countries, where resources for such actions are limited.

Greater research needs to be directed towards understanding the nature of regional climates, hydrological regimes, geological processes, including their variability and potential for change. Such knowledge is essential in order to estimate the potential frequency of natural hazards and the options for adaptation that are available. Early warning is effective only if it reaches the affected people who have to respond to such a situation. Science and technology should try to break the barrier of financial resources to enable such warning to reach even the remotest corners of the affected areas.

Field studies are probably the most difficult and costly tasks within this integrated approach. The difficulties encountered are connected to the complexity of the phenomena investigated and the difficulty of direct observation. The exceptional conditions in which such extreme events occur do not generally permit a sufficient number of observations for the same type of territorial reality to work out the peculiar behavioural laws for that area. 
Reference to different territorial situations also highlights another problem: that of the homogeneity of data given the substantial territorial peculiarity in which the phenomena occur.

In relation with this Special Session, large woody debris accumulations, debris and hyper-concentrated flows are a familiar hazard in mountain areas and regularly cause both loss of life, livestock and property and disruption of communications. The potential for such losses is growing as the mountain areas are increasingly developed and insurance claims, as a result of this threat, are steadily rising. Further, the development itself (e.g. construction of roads and recreational areas, tourist trade) can increase the incidence of LWD, hyperconcentrated and debris flows by changing their topographic, features and soil and vegetation controls. Hazard assessment is therefore increasingly required in land use planning in mountain environments.

The ideal sequence that should be pursued in the approach to the difficult problem of the management and hazard mitigation of LWD, hyper-concentrated and debris flows can be outlined as follows:

1) first, a systematic collection of field data should be carried out in order to provide a large base of reliable data that could allow a better knowledge of the existing risk trends and a deeper understanding of the mechanics of the phenomena, along with their general behaviour and effects;

2) secondly, effective mathematical models, which strongly depend on data and measurements collected and performed in the field for their calibration and design, should be constantly developed, updated when needed, tested and applied;

3) hazard mapping techniques and identification of possible scenarios, which need reliable models to be effective and sound, should then be set up;

4) on the basis of the knowledge achieved in the previous steps, the best mitigation solutions should be identified, designed and built up;

5) finally a program of systematic observations on the sites, where risk has been mitigated, should be planned and carried out to detect any shortcoming and test the efficiency of the investigations.

Each of the above studies and investigations needs improvements and depends, to achieve them, on improvements in the other fields. As an example, current monitoring procedures need, in general, to be improved to be able to perform measurements in all the different field conditions. Improving measurement and documentation procedures would provide a better knowledge and ideas for new and more advanced models. The application of existing models based on the data collected in the field and the development of reliable new ones would allow, on one hand, to better focus what to observe in the field and, on the other hand, improve both mitigation methodologies and measures and hazard mapping procedures. The field application of these latter would then identify new parameters to be measured and introduced in the models. From all these activities would emerge the best mitigating solutions to be applied.

In this Special Session, particular attention will be given to both field data acquisition-monitoring approaches for sediment transport, LWD, hyper- 
concentrated and debris flows, and to the modelling and hazard assessment. Also the discussion of case study of catchments located in Italy and Southern Andes (Chile and Argentina) will be part of the Session.

\section{References}

[1] Lorenzini, G. and Mazza M. 2004 Debris Flows. Phenomenology and Rheological Modelling.Wit press, Southampton, UK

[2] $\quad 4^{\text {th }}$ World Water Forum 2006 -Framework theme 5

[3] Meadcroft et al .1996. Development of new risk assessment procedures for coastal structures. In Advances in coastal structures and breakwaters. T. Thelford (Editor), London, UK

[4] International Strategy for Disaster Reduction (ISDR) 2004. Terminology: Basic terms of disaster reduction

D. De Wrachien

M. A. Lenzi 